\section{MS7-P2 Structural and biochemical insights into grainyhead-like 1 and its homolog grainyhead-like 3}

Qianqian Ming ${ }^{1}$, Yvette Roske ${ }^{1}$, Anja Schuetz ${ }^{1}$, Udo Heinemann ${ }^{1}$

1. Max-Delbrück Center for Molecular Medicine Berlin-Buch, Germany

email: Qianqian.Ming@mdc-berlin.de

Grainyhead is one of the best studied genes encoding transcription factors of the CP2 family (TFCP2) which contribute to the regulation of gene expression from early embryonic develop $\neg$ ment to terminal cell differentiation in Drosophila. It has been established that there are three Grainyhead homologs in the human genome, encoding transcription factors Grainyhead-like 1, 2, and 3 (Grhl1, Grhl2, Grhl3). They are expressed in the surface ectoderm and in other epithelial tissues. By regulating genes involved in cell junction formation and proliferation, they can control the development and differentiation of multicellular epithelia .Molecular mechanisms of Grhl function have been ascribed in three ways. Grhl genes can be regulated at the transcriptional level or by alternative splicing . Following post-translational modification, Grhl proteins can compete with an activator or repressor for DNA binding or associate with other transcription factors, cooperatively regulating common downstream genes. Despite the experimental studies already been published, the knowledge on the structural mechanism of activity of this transcription factors remains sparse. There is no structure report about any $\mathrm{CP} 2$ family member as yet of. In our current work we employ X-ray crystallography to analyze the three-dimensional structure of human Grhl1 and Grhl3. Like all members of the Grhl family the proteins Grhl1-3 are composed of three domains, a N-terminal transactivation domain (TAD), a central DNA-binding domain (DBD) and a C-terminal dimerization domain (DD). We have obtained soluble Grhl1 and Grhl3 protein with several constructs varying in length, and we obtained crystals for truncated Grhll. The crystal optimization and structure determination is ongoing, and we hope to present a first glimpse of the 3D structure at the conference. Meanwhile we used electrophoretic mobility shift assays (EMSA) and isothermal titration calorimetry (ITC) to investigate the DNA binding behavior of the soluble Grhl constructs. In the future, upon structural determination of the Grhl1 DBD by itself and in complex with DNA response elements, as well as by comparison of the Grhl1 and Grhl3 DNA-binding network, we aim to explain the structural basis of the Grhl proteins' DNA sequence recognition and binding to the response elements.

Keywords: cp2,grhl,transcription factor,crystal

\section{MS7-P3 Fascinating novel coordination chemistry of copper(II) bound to d(CGCGCG) and explored by single crystal $\mathrm{X}$-ray analysis \\ Bernhard Spingler ${ }^{1}$, Melanie Rohner ${ }^{1}$, Alfredo Medina-Molner ${ }^{1}$}

1. University of Zurich, Switzerland

email: spingler@chem.uzh.ch

Generating supramolecular $n$-dimensional arrays by combining metals with DNA building blocks that are modified in order to include metal binding sites is a very active field of research ${ }^{(1)}$. Using unmodified nucleobases avoids a potentially difficult synthesis with the disadvantage of a less defined coordination mode of the metal. In the following, we will concentrate on copper(II) as a linking metal and guanine as the natural ligand for metals in unmodified DNA. In this context, it is interesting to explore the binding of two guanines, that are part of two different oligonucleotides, to one copper center as a simple way in order to build up supramolecular structures.

Previously, we were exploring the ability of mono- and dinuclear metal complexes to induce Z-DNA ${ }^{(2)}$. In this presentation, we will report about our X-ray studies of copper(II) ions with DNA hexamers ${ }^{(3)}$ of the general sequence $\mathrm{d}(\mathrm{CG})_{3}$ that form 3 different packing modes as observed in 3 crystal structures with resolutions ranging from $2.15 \AA$ to $1.45 \AA$. Dependent upon other cations being present and/or crystallization conditions, different packing motives were observed. Excitingly, we observed the first intramolecular O6,N7-chelate of a neutral purine nucleobase to copper as well as the first meridional N,N,O coordination of 2 guanines to copper. The fascinating coordination chemistry of copper(II) imposed by the Z-DNA oligonucleotides and its differences to simple nucleobases as ligands for copper(II) ${ }^{(4)}$ will be discussed as well.

(1) Burns, J. R.; Zekonyte, J.; Siligardi, G.; Hussain, R.; Stulz, E. Molecules 2011, 16, 4912; McLaughlin, C. K.; Hamblin, G. D.; Sleiman, H. F. Chem. Soc. Rev. 2011, 40, 5647; Kalachova, L.; Pohl, R.; Bednarova, L.; Fanfrlik, J.; Hocek, M. Org. Biomol. Chem. 2013, 11, 78.

(2) Medina-Molner, A.; Spingler, B. Chem. Commun. 2012, 48, 1961; Medina-Molner, A.; Rohner, M.; Pandiarajan, D.; Spingler, B. Dalton Trans. 2015, 44, 3664.

(3) Kagawa, T. F.; Geierstanger, B. H.; Wang, A. H.-J.; Ho, P. S. J. Biol. Chem. 1991, 266, 20175; Geierstanger, B. H.; Kagawa, T. F.; Chen, S.-L.; Quigley, G. J.; Ho, P. S. J. Biol. Chem. 1991, 266, 20185; Rohner, M.; Medina-Molner, A.; Spingler, B. 2015, submitted.

(4) Sletten, E.; Flogstad, N. Acta Cryst. 1976, B32, 461; Sheldrick, W. S. Acta Cryst. 1981, B37, 1820; Zhou, P.; Li, H. Dalton Trans. 2011, 40, 4834; Nagapradeep, N.; Venkatesh, V.; Tripathi, S. K.; Verma, S. Dalton Trans. 2014, 43, 1744. 


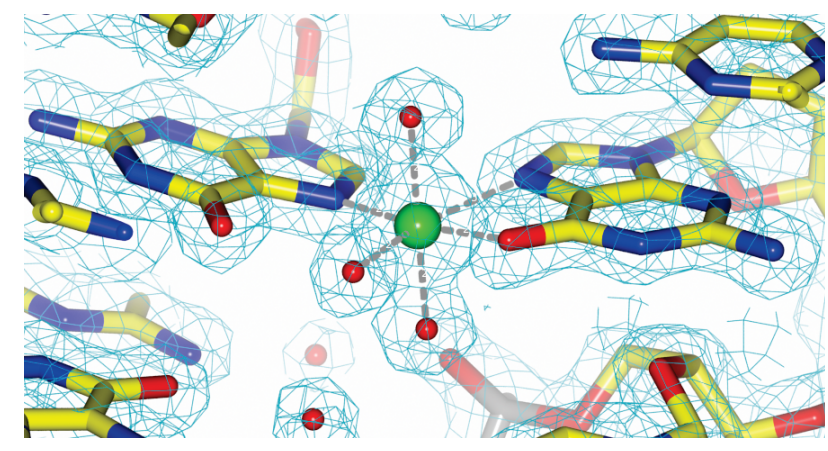

Figure 1. Intramolecular O6,N7-chelate of guanine (right) to copper (green) and meridional N,N,O coordination of 2 guanines to copper.

Keywords: DNA, metal, supramolecular arrangement, copper(II)

\section{MS7-P4 Signal sequence binding to the archaeal signal recognition particle \\ Elisabeth Sauer-Eriksson ${ }^{1}$, Tobias Hainzl ${ }^{1}$}

1. Department of Chemistry, Umea University, Sweden

email: elisabeth.sauer-eriksson@umu.se

The signal recognition particle (SRP) co-translationally targets proteins to the endoplasmic reticulum in eurkaryotes or to the plasma membrane in prokaryotes. As the initiating step, SRP binds to the N-terminal signal sequence of nascent secretory or membrane proteins as they emerge from the ribosome. The SRP-ribosome nascent chain complex is then targeted to the membrane through a GTP-dependent interaction with the SRP receptor (SR). The signal sequence is released from SRP and inserted into the translocon channel. Finally, GTP hydrolysis triggers the dissociation of SRP from SR, and SRP can start another cycle of protein targeting.

SRP composition varies in the three domains of life. However, the evolutionary conserved SRP core only comprises the SRP54 protein bound to the coaxial stacked helices 5 and 8 of SRP RNA. SRP54 comprises an N-terminal NG domain that interacts with SR and a $\mathrm{C}$-terminal methionine-rich $\mathrm{M}$ domain that binds to the signal sequence. Signal-sequence binding in the SRP54 M domain must therefore be effectively communicated to the SRP54 NG domain for receptor interaction. We have determined the $2.9 \AA$ crystal structure of unbound- and signal-sequence bound SRP forms, both present in the asymmetric unit. The structures provide evidence for a coupled binding and folding mechanism in which signal-sequence binding induces the concerted folding of the GM linker helix, the finger loop, and the C-terminal alpha helix aM6. This mechanism allows for a high degree of structural adaptability of the binding site and suggests how signal-sequence binding in the $\mathrm{M}$ domain is coupled to repositioning of the NG domain for accelerated receptor interaction. We propose that signal-sequence recognition via a disorder-to-order transition of multiple structural elements facilitates specific recognition of widely diverse signal sequences.

Keywords: signal recognition particle, SRP, crystal structure, signal peptide, archaea 